\title{
Delayed contrast enhancement cardiac magnetic resonance imaging in trastuzumab induced cardiomyopathy
} Nazanin Fallah-Rad ${ }^{1}$, Matthew Lytwyn ${ }^{1}$, Tielan Fang1, Iain Kirkpatrick ${ }^{3}$ and Davinder S Jassal*1,2,3

Address: ${ }^{1}$ Institute of Cardiovascular Sciences, St. Boniface Research Centre, University of Manitoba, Winnipeg, Manitoba, Canada, ${ }^{2}$ Section of Cardiology, Department of Cardiac Sciences, University of Manitoba, Winnipeg, Manitoba, Canada and ${ }^{3}$ Department of Radiology, University of Manitoba, Winnipeg, Manitoba, Canada

Email: Nazanin Fallah-Rad - umfallah@hotmail.com; Matthew Lytwyn - mlytwyn@sbrc.ca; Tielan Fang - tfang@sbrc.ca; Iain Kirkpatrick - ikirkpatrick@sbgh.mb.ca; Davinder S Jassal* - djassal@sbgh.mb.ca

* Corresponding author

Published: 22 January 2008

Journal of Cardiovascular Magnetic Resonance 2008, 10:5 doi:10.1 186/1532-429X-10-5
Received: 18 December 2007

Accepted: 22 January 2008

This article is available from: http://www.jcmr-online.com/content/ $10 / 1 / 5$

(c) 2008 Fallah-Rad et al; licensee BioMed Central Ltd.

This is an Open Access article distributed under the terms of the Creative Commons Attribution License (http://creativecommons.org/licenses/by/2.0), which permits unrestricted use, distribution, and reproduction in any medium, provided the original work is properly cited.

\begin{abstract}
Background: Trastuzumab (Herceptin), an antagonist to the human epidermal growth factor 2 (HER2) receptor significantly decreases the rates of breast cancer recurrence and mortality by $50 \%$. Despite therapeutic benefits, the risk of cardiotoxicity with trastuzumab ranges from $10-15 \%$ when administered sequentially following anthraycline chemotherapy. Little is known about the utility of cardiac magnetic resonance (CMR) in the assessment of trastuzumab mediated cardiomyopathy.

Methods and results: Between 2005-2006 inclusive, 160 breast cancer patients were identified at a single tertiary care oncology centre. Of the total population, 10 patients (mean age $40 \pm 8$ years) were identified with trastuzumab induced cardiomyopathy, based on a LVEF less than $40 \%$ on serial MUGA or echocardiography. CMR was performed in all patients to determine LV volumes, systolic function and evidence of late gadolinium enhancement (LGE). At the time of diagnosis of trastuzumab induced cardiomyopathy, the mean LVEF was $29 \pm 4 \%$. Subepicardial linear LGE was present in the lateral portion of the left ventricles in all 10 patients.
\end{abstract}

Conclusion: LGE-CMR is a novel way of detecting early changes in the myocardium due to trastuzumab induced cardiotoxicity.

\section{Introduction}

Breast cancer is a major public health concern that affects 1 in 7 women in their lifetime [1]. Anthracyclines are commonly used in the setting of adjuvant therapy in the treatment of breast cancer patients. While anthracyclines significantly improve clinical morbidity and mortality, there are notable cardiotoxic side effects [2]. Recent understanding of the biology of breast cancer has lead to the introduction of a new therapeutic agent, Trastuzumab
(Herceptin), an antagonist to the human epidermal growth factor 2 (HER2) receptor, which is found in $25 \%$ of breast cancer patients [3]. When added to conventional anthracycline chemotherapy, trastuzumab significantly decreases the rates of recurrence and mortality by $50 \%$ in HER-2 positive breast cancer patients [4-6]. Despite therapeutic benefits however, the risk of cardiotoxicity with trastuzumab ranges from $10-15 \%$ when administered in combination with anthracyline therapy $[7,8]$. 
Serial multiple gated acquisition scans (MUGA) are widely used to monitor cardiac dysfunction in breast cancer patients. However, with the improvement in both spatial and temporal resolution of cardiac magnetic resonance (CMR) over the past decade, it has now become the gold standard for the non-invasive assessment of left ventricular (LV) systolic dysfunction. Additionally, late gadolinium enhancement (LGE) can detect myocardial scarring. Although frequently used in the assessment of dilated cardiomyopathies secondary to ischemia or myocarditis [9], little is known about the utility of CMR in the assessment of trastuzumab induced cardiomyopathy. We report a case series of trastuzumab induced myocarditis characterized by left ventricular dysfunction and focal epicardial LGE using CMR imaging.

\section{Methodology \\ Patient population}

Between 2005-2006 inclusive, 160 breast cancer patients who received trastuzamab in addition to anthracyline based adjuvant therapy were identified at a tertiary care oncology centre. All patients received FEC (5-fluorouracil, epirubicin and cyclophoshamide) for a total of 6 cycles. The mean duration between completion of chemotherapy and initiation of trastuzumab was $2 \pm 1$ months. Of the total population, 10 patients were identified with trastuzumab induced cardiomyopathy based on LV ejection fraction less than $40 \%$ on either serial MUGA or echocardiography. The medical records of all 10 patients were extensively reviewed for baseline demographic data. The retrospective study was approved by the local institutional review board.

\section{Cardiac MRI}

CMR was performed on all 10 patients using a $1.5 \mathrm{~T}$ scanner (Avanto, Siemens, Erlangen, Germany). Morphologic images in the cardiac short axis, 4 chamber long axis and 2 chamber long axis planes were acquired using IR-prepared dark blood HASTE sequences (TR $600 \mathrm{~ms}$, TE 26 $\mathrm{ms}, 6 \mathrm{~mm}$ slice thickness, $1.8 \mathrm{~mm}$ interslice gap). In the same planes, cine-CMR was performed using a breathhold balanced steady state free precession sequence (TrueFISP, TR $42 \mathrm{~ms}$, TE $1.2 \mathrm{~ms}$, FA $70^{\circ}, 6 \mathrm{~mm}$ slice thickness, matrix $192 \times 174)$. The cine-CMR short-axis images encompassed the entire LV from the base to the apex (stack of 10 sequential short-axis slices; TR $64 \mathrm{~ms}$, TE 1 $\mathrm{ms}$, FA $80^{\circ}, 8 \mathrm{~mm}$ slice thickness, $1.6 \mathrm{~mm}$ interslice gap, matrix $192 \times 132$ ) to obtain a left ventricular ejection fraction (LVEF). Late gadolinium enhancement was performed after 10 minutes of $0.2 \mathrm{mmol} / \mathrm{kg}$ injection of Gadolinium (Gd-DTPA, Magnevist, Schering, Germany) using a T1-weighted IR-prepared multislice TrueFISP sequence with magnitude and phase sensitive reconstruction. Images were acquired sequentially in the short axis, followed by horizontal and vertical long axis images (TR $700 \mathrm{~ms}$, TE $1.0 \mathrm{~ms}, \mathrm{FA} 40^{\circ}, 8 \mathrm{~mm}$ slice thickness, $1.6 \mathrm{~mm}$ interslice gap, matrix $192 \times 144$ ).

\section{Results}

The total population included 10 patients (mean age $40 \pm$ 8 years, range 27 to 56 years) with normal LVEF at baseline and preserved systolic function following administration of anthracycline based chemotherapy using serial MUGA scans (Table 1). Cardiovascular risk factors, dose and frequency of chemotherapy, concomitant use of radiation therapy, and duration of trastuzumab therapy (3-5 months) were similar in the entire patient cohort (Table $1)$.

At the time of diagnosis of trastuzumab induced cardiomyopathy, the left ventricular cavities were dilated with moderate to severe global LV systolic dysfunction on CMR (Table 2). The mean LVEF was for the total population was $29 \pm 4 \%$ (Table 2). Subepicardial linear LGE was present in the lateral portion of the left ventricles in all 10 patients suggesting the presence of trastuzumab induced myocarditis (Figure 1).

Following the discontinuation of trastuzumab, 6 patients have recovered normal LV systolic function, while 4

Table I: Clinical Characteristics of Patient Population $(n=10)$

\begin{tabular}{ccccccc}
\hline Case No. & Age & CV risk & Radiotherapy & Baseline LVEF (\%) & Post Chemothx LVEF (\%) & Trastuzumab Duration \\
\hline 1 & 33 & None & Yes & 55 & 52 & 4 months \\
2 & 41 & HTN & Yes & 60 & 58 & 5 months \\
3 & 27 & None & Yes & 54 & 56 & 4 months \\
4 & 39 & None & Yes & 65 & 62 & 4 months \\
5 & 44 & Lipids & Yes & 58 & 55 & 5 months \\
6 & 38 & None & Yes & 56 & 52 & 3 months \\
7 & 56 & HTN & Yes & 60 & 62 & 4 months \\
8 & 45 & HTN & Yes & 54 & 55 & 5 months \\
9 & 32 & None & Yes & 55 & 58 & 4 months \\
10 & 40 & HTN & Yes & 60 & 5 & \\
\hline
\end{tabular}

CV, cardiovascular; LVEF, left ventricular ejection fraction; chemothx, chemotherapy; HTN, hypertension. 
Table 2: CMR findings of patient population $(n=10)$

\begin{tabular}{cccccc}
\hline Case No. & Age & LVEF (\%) & Delayed enhancement & Medical treatment & 6 month flu LVEF (\%) \\
\hline I & 33 & 32 & Lateral, septal & ACEl, beta blockers & 55 \\
2 & 41 & 28 & Lateral & ACEl, beta blockers & 60 \\
3 & 27 & 30 & Lateral & ACEl, beta blockers & 40 \\
4 & 39 & 25 & Lateral & ACEl, beta blockers & 65 \\
5 & 44 & 35 & Lateral, septal & ACEl, beta blockers & 60 \\
6 & 38 & 30 & Lateral & ACEl, beta blockers & 40 \\
7 & 56 & 25 & Lateral & ACEl, beta blockers & 30 \\
8 & 45 & 24 & Lateral & ACEl, beta blockers & 55 \\
9 & 32 & 30 & Lateral & ACEl, beta blockers & 35 \\
10 & 40 & 34 & Lateral & 58 \\
\hline
\end{tabular}

LVEF, left ventricular ejection fraction; $A C E l$, angiotensin converting enzyme inhibitor.

patients have persistent LV dysfunction at six month followup, despite appropriate heart failure medications including ACE inhibition and beta blockade (Table 2). The linear LGE-CMR findings persisted at 6 months in all patients despite improvement in LVEF in over half of the patients.

\section{Discussion}

An increasing understanding of the biology of breast cancer has lead to the identification of novel therapeutic targets. The HER2 receptor is a member of the epidermal growth factor receptor family of transmembrane tyrosine kinases and is normally involved in the regulation of cell proliferation. Increased expression of HER2 is detected in $25-30 \%$ of breast cancers and is associated with poorly differentiated tumors with a high proliferative rate, posi-

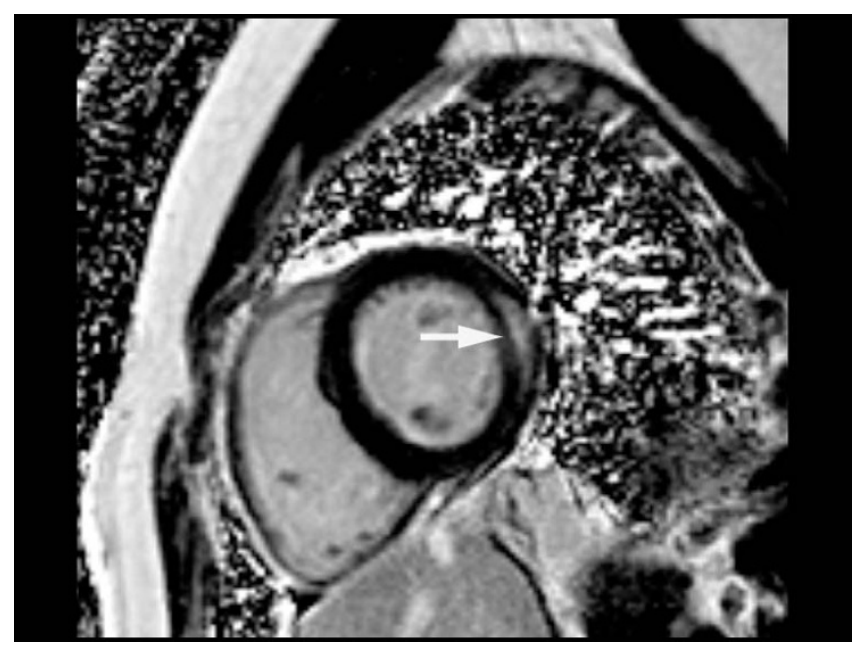

\section{Figure I}

Short axis phase sensitive reconstructed IR-TrueFISP image through the mid-ventricle demonstrates subepicardial linear late gadolinium enhancement (arrow) in the lateral wall of a patient who had received Trastuzumab. tive axially lymph nodes and decreased expression of estrogen and progesterone receptors [3]. These characteristics are associated with an increased risk of disease recurrence and death due to breast cancer [3].

Trastuzumab (Herceptin) is a relatively new chimerized monoclonal antibody that targets the extracellular portion of the HER2 membrane protein. Previous studies have demonstrated that trastuzumab provides considerable therapeutic benefits, when added to conventional anthracycline chemotherapy, in decreasing the rates of disease recurrence and death in HER2 overexpressed metastatic breast cancers [4-6]. Despite its therapeutic benefit, trastuzumab is cardiotoxic with a $5 \%$ prevalence of cardiomyopathy when used as monotherapy and 10-15\% prevalence of cardiomyopathy when used in combination with anthracyclines $[7,8]$.

Various theories have been suggested regarding the possible pathogenetic origin of trastuzumab induced cardiomyopathy. These include potentiation of anthracycline induced cardiotoxicity and immune mediated destruction of cardiomyocytes [10]. There is increasing evidence as well supporting a direct toxic effect of HER2 blockade on the myocardial tissue. HER2 signaling appears to play an important role in the embryonic cardiac development and cardioprotection; the blockade of this pathway by trastuzumab can also lead to myocardial inflammation and damage [11].

The use of CMR for the non-invasive characterization of trastuzumab induced myocarditis is novel. Whereas CMR has become the standard diagnostic test in the evaluation of suspected myocarditis $[9,12]$, to date, there are no reports in the literature describing the utility of LGE for the diagnosis of trastuzumab induced cardiomyopathy. The current report demonstrated the common finding of LGE of the subepicardial lateral wall in all patients, which may be a typical distribution and location of myocarditis 
in this drug induced cardiomyopathy. Although 6 patients recovered LVEF by CMR with appropriate therapy for CHF, it is of interest that the LGE findings persisted up to 6 months in all patients, suggesting persistent injury to the myocardium. A limitation of the current retrospective study however is the small number of individuals. A larger prospective series may enable us to make more substantive conclusions regarding the role of LGE-CMR in the diagnosis and prognosis of this patient population.

\section{Conclusion}

Late gadolinium enhancement using CMR is a novel way of detecting early changes in the myocardium due to trastuzumab induced cardiotoxicity. Future studies are required to validate identification of positive delayed enhancement using CMR as a subclinical marker for future LV dysfunction in this select population. Early detection of LV dysfunction using LGE-CMR may allow one to adjust treatment with trastuzumab prior to the development of irreversible heart failure.

\section{Competing interests}

The author(s) declare that they have no competing interests.

\section{References}

I. Armstrong K, Eisen A, Weber B: Primary care: assessing the risk of breast cancer. N Engl J Med 2000, 342:564-57I.

2. Barry E, Alvarez J, Miller T, Lipshultz S: Anthracycline-induced cardiotoxicity: course, pathophysiology, prevention and management. Expert Opinion 2007, 8:1039-1058.

3. Slamon DJ, Clark GM, Wong SG, Levin WJ, Ullrich A, McGuire WL: Human breast cancer: correlation of relapse and survival with amplication of the HER-2/neu oncogene. Science 1987, 235:244-707-12.

4. Slamon DJ, Leland-Jones B, Shak S, Fuchs SH, Paton V, Bajamonder A, Fleming T, Eiermann W, Wolter J, Pegram M, Baselga J, Norton L: Use of chemotherapy plus a monoclonal antibody against HER2 for metastatic breast cancer that overexpresses HER2. N Engl J Med 200I, 344:783-92.

5. Romond EH, Perez EA, Bryant J, Suman VJ, Geyer CE Jr, Davidson NE, Tan-Chiu E, Martino S, Paik S, Kaufman PA, Swain SM, Pisansky TM, Fehrenbacher L, Kutteh LA, Vogel VG, Visscher DW, Yothers G, Jenkins RB, Brown AM, Dakhil SR, Mamounas EP, Lingle WL, Klein PM, Ingle JN, Wolmark N: Trastuzumab plus adjuvant chemotherapy for operable HER2-positive breast cancer. $N$ Engl J Med 2005, 353:1673-84.

6. Piccart-Gebhart MJ, Procer M, Leyland-Jones B, Goldhirsch A, Untch M, Smith I, Gianni L, Basela J, Bell R, Jackisch C, Cameron D, Dowsett M, Barrios CH, Steger G, Huang CS, Anderson M, Inbar M, Lichinitser M, Lang I, Nutz U, lawata H, Thomssen C, Lohrisch C, Suter TM, Ruschoff J, Suto T, Greatorex V, Ward C, Straehle C, McFadden E, Dolci MS, Gelber RD: Trasuzumab after adjunctive chemotherapy in HER2-positive breast cancer. N Engl J Med 2005, 353:1658-72.

7. Ewer MS, Gibbs HR, Swafford J, Benjamin RS: Cardiotoxicity in patients receiving trastuzumab (Herceptin): primary toxicity, synergistic or sequential stress, or surveillance artifact? Semin Oncol 1999, 26(Suppl I 2):96-101.

8. Tan-Chiu E, Yothers G, Romond E, Geyer CE Jr, Ewer M, Leefe D, Shannon RP, Swain SM, Brown A, Fehrenbacher L, Vogel VG, Seay TE, Rastogi P, Mamounas EP, Wolmark N, Bryant J: Assessment of cardiac dysfunction in a randomized trial comparing doxrobucin and cyclophosphamide followed by paclitaxel, with or without trastuzumab as adjuvant therapy in nodepositive, human epidermal growth factor receptor 2-overex- pressing breast cancer- NSABP B-3I. I Clin Oncol 2005 23:78। I-9.

9. Skouri HN, Dec GW, Friedrich MG, Cooper LT: Noninvasive imaging in myocarditis. J Am Coll Cardiol 2006, 48:2085-93.

10. Chien KR: Herceptin and the heart-a molecular modified of cardiac failure. $N$ Engl J Med 2006, 354:789-790.

II. Erickson SL, O'Shea KS, Ghaboosi N, et al.: ErbB3 is required for normal cerebellar and cardiac development: a comparison with ErbB2- and heregulin-deficient mice. Development 1997, I 24:4999-50I I.

12. Dill T, Ekinci O, Hansel J, Kluge A, Briedenbach C, Hamm CW: Delayed contrast-enhanced magnetic resonance imaging for the detection of autoimmune myocarditis and long-term follow-up. J Cardiovasc Magn Reson 2005, 7(2):52 I-3.
Publish with Biomed Central and every scientist can read your work free of charge

"BioMed Central will be the most significant development for disseminating the results of biomedical research in our lifetime. "

Sir Paul Nurse, Cancer Research UK

Your research papers will be:

- available free of charge to the entire biomedical community

- peer reviewed and published immediately upon acceptance

- cited in PubMed and archived on PubMed Central

- yours - you keep the copyright

Submit your manuscript here:

http://www.biomedcentral.com/info/publishing_adv.asp
BioMedcentral 